\title{
Elektrokardiyografi Sinyali ile Bazal Metabolizma Hızının Cinsiyet Bazlı Yapay Zekâ Tabanlı Tespiti
}

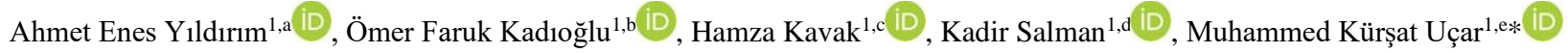 \\ Zeliha Uçar ${ }^{2}$ iD, Mehmet Recep Bozkurt ${ }^{1, \mathrm{f}}$ \\ ${ }^{1}$ Sakarya Üniversitesi, Mühendislik Fakültesi, Elektrik-Elektronik Mühendisliği Bölümü, Sakarya, Türkiye

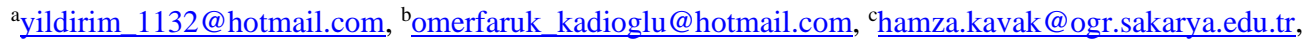 \\ ${ }^{\mathrm{d} k \text { kadir.salman@ogr.sakarya.edu.tr, }}{ }^{\mathrm{e} \text { mucar@ } @ \text { sakarya.edu.tr, }},{ }^{\mathrm{f} \text { mbozkurt@ sakarya.edu.tr }}$ \\ ${ }^{2}$ İstanbul Okan Üniversitesi, Sağlık Bilimleri Enstitüsü, Beslenme ve Diyetetik Bölümü, İstanbul, Türkiye \\ zelihaguvenc@hotmail.com
}

\section{$\ddot{O} \mathbf{z}$}

Bazal Metabolizma Hızı (BMH) günlük harcanan ve alınması gereken enerji hakkında bilinmesi gereken en önemli unsurlardan biridir. Literatürde genellikle kalorimetreler ve birtakım denklemler tarafından tespit edilmektedir. Bu çalışmada BMH tahmini için elektrokardiyografi (ECG) sinyalleri ile yapay zekâ tabanlı bir model oluşturulmuştur. Öncelikle bireylerden toplanan ECG sinyalleri gürültülerden temizlenip filttrelenmiştir. Daha sonra özellik çıkartılıp özellik seçme algoritmaları yardımıyla azaltılmıştır. Elde kalan özelliklerle yapay zekâ algoritmaları sayesinde BMH tahmininde bulunulmuştur. Erkekler için $\mathrm{R}=0.91$, kadınlar için $\mathrm{R}=0.99$ değerlerine sahip modeller oluşturulmuştur. Performans değerlendirme kriterleri de göz önüne alınarak en iyi model kadınlar için de erkekler için de Linear Regression modeli seçilmiştir. Tüm bu sonuçlara bakıldığında günlük hayatta BMH tahmini için önerilen modelin kullanılabileceği belirlenmiştir.

Anahtar kelimeler: Yapay Zekâ, Bazal Metabolizma Hızı, Elektrokardiyografi Sinyali

\section{Gender-Based Artificial Intelligence Based Detection of Basal Metabolic Rate by Electrocardiography Signal}

\begin{abstract}
Basal Metabolic Rate (BMR) is one of the most important factors that should be known about the energy consumed and taken daily. It is usually determined by calorimeters and some equations in the literature. In this study, an artificial intelligence-based model was created with electrocardiography (ECG) signals for BMR prediction. First of all, ECG signals collected from individuals are cleaned from noise and filtered. Later, the feature was removed and reduced with the help of feature selection algorithms. BMR predictions have been made with the remaining features thanks to artificial intelligence algorithms. Models with $R=0.91$ for men and $R=0.99$ for women were created. Considering the performance evaluation criteria, the Linear Regression model was chosen as the best model for both women and men. Considering all these results, it was determined that the proposed model could be used for BMR estimation in daily life.
\end{abstract}

Keywords: Artificial Intelligence, Basal Metabolic Rate, Electrocardiography Signal 


\section{Giriş (Introduction)}

Bazal Metabolizma Hızı (BMH) genellikle yaşamı sürdürmek için gerekli temel süreçlerle uyumlu minimum metabolizma hizı olarak kabul edilir (Speakman, Król, and Johnson 2004). Günlük harcanan ve alınması gereken enerji hakkında bilinmesi gereken en önemli parametrelerden biridir. Ancak günlük hayatta ölçümünü yapmak oldukça zahmetli ve maliyetli bir iştir. Literatürde kullanılan en yaygın BMH ölçüm yöntemi dolaylı kalorimetredir. Dolaylı kalorimetre, oksijen tüketimi ve karbondioksit üretimi ölçümlerinden bazal metabolizma hızını tespit eder (Ferrannini 1988).

Bir diğer BMH tespiti için kullanılan yöntem de BMH denklemleridir (Müller et al. 2001). Bu denklemlerin BMH tespitinde kullanılması oldukça pratiktir. Ancak yapılan araştırmalar gösteriyor ki denklemlerin sonucunda elde edilen değerler, gerçek BMH değerlerinden oldukça farklıdır (Flancbaum et al. 1999). Tüm bu durumlar göz önüne alındığında BMH tespiti için yeni yöntemlere ihtiyaç duyulduğu gözlemlenmiştir.

$\mathrm{Bu}$ çalışmanın amacı maliyetli ve ölçümü zor olan yöntemler yerine yapay zekâ tabanlı güvenilir yeni bir yöntem geliştirmektir. $\mathrm{Bu}$ çalışmada, literatürdeki yöntemlerin aksine kalorimetreler yerine elektrokardiyografi (ECG) sinyalleri kullanarak BMH tahmini yapmaktır. ECG sinyali kalbin elektriksel faaliyeti sonucu oluşan ve deriden elektrotla ölçülebilen biyopotansiyel sinyallerdir (Nur Göz et al. 2017). Bu çalışmada 324 farklı kişiden ECG sinyalleri alınarak 3 farklı filtreleme sonucunda gürültüleri en aza indirilmiş bir sinyal elde edilmiştir. Bu sinyalden çıkarılan 23 özelliğe demografik bilgiler de eklenerek toplamda 27 özellik elde edilmiştir. Spearman özellik seçme algoritması yardımıyla makine öğrenmesinde kullanılacak özellikler belirlenmiştir. Makine öğrenmesi algoritmaları kullanılarak seçilen özellikler yardımıyla BMH tahmini yapılmıştır ve performans değerlendirme kriterleri göz önüne alınarak en iyi sonuç veren model belirlenmiştir. Bütün süreç kadın ve erkek için ayrı ayrı tekrar edilmiştir ve cinsiyet bazlı modeller geliştirilmiştir.

Bu çalışma literatürle kıyaslandığında birçok yenilik içermektedir. (1) Literatürde BMH hesaplamaları için denklemler kullanılırken, bu çalışmada yapay zekâ tabanlı algoritmalar kullanılmıştır. (2) Ölçümlerde zahmetli ve maliyetli kalorimetreler kullanılırken bu çalışmada ECG sinyalleri kullanılmıştır. (3) Özellik seçme algoritmaları kullanılarak modelin doğruluk oranları arttırılmıştır ve güvenilirliği yüksek bir tahmin modeli oluşturulmuştur. Tüm bu çalışmalar göz önüne alındığında literatüre yenilik katmaktadır.

Çalışmada kadın/erkek/kadın-erkek olmak üzere 3 farklı model geliştirilmiştir. Erkekler için geliştirilen model $\mathrm{R}=0.91$, kadınlar için $\mathrm{R}=0.99$, kadın-erkek için $\mathrm{R}=0.87$ değerlerine sahiptir. Sonuçlar göz önüne alındığında cinsiyet bazlı oluşturulan modellerin doğruluk oranlarının daha yüksek olduğu gözlemlenmiştir.

\section{Materyal ve Yöntem (Material and Method)}

Araştırmada izlenen yol şu şekilde açıklanabilir (Şekil 1). İlk olarak bireylerden alınan ECG sinyalleri filtrelenmiştir ve bu filtrelenmiş sinyallerden özellik çıkarımı yapılmıștır. Daha sonra bu özelliklere demografik bilgiler eklenmiştir. Bir sonraki adımda özellik seçme algoritması kullanılarak özellik sayısı azaltılmıştır. Son olarak bu özelliklerden makine öğrenmesi algoritmaları sayesinde BMH tahmini yapılmıştır.

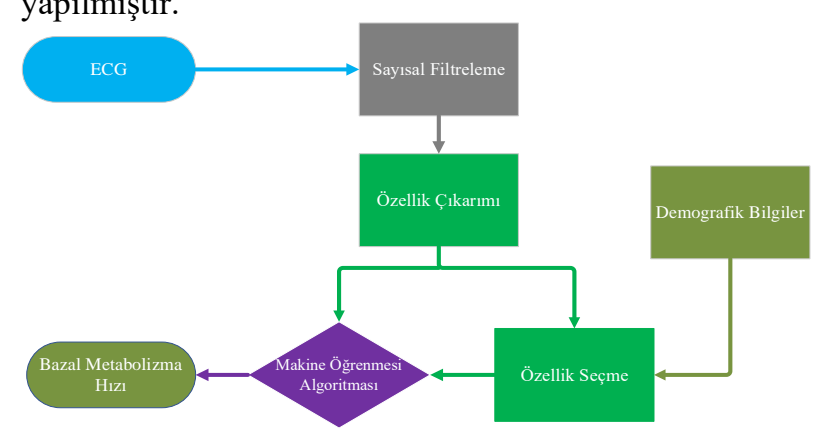

Şekil 1. Çalışma akış diyagramı (work flow diagram)

\subsection{Verilerin toplanmasl (Data collection)}

Bu çalışmada kullanılan veriler Sakarya Üniversitesi Sağlıklı Beslenme ve Danışmanlık Birimi'ne beslenme ve danışmanlık hizmeti için Eylül 2019 - Şubat 2020 tarihleri arasında başvuru yapan gönüllülerden alınmıştır. Çalışmanın yapılabilmesi için gerekli etik kurul onay ve veri kullanım izni alınmıştır.

Çalışmada 324 bireyden alınan ECG sinyali ve BMH verileri toplanmıştır. Bu veriler ve sinyaller kullanılarak BMH'nın yüksek doğruluk oranında tahmin edilmesi amaçlanmaktadır.

Cinsiyete göre demografik bilgiler ve $\mathrm{BMH}$ dağılımları farklılık gösterdiği için BMH'nın cinsiyet bazlı hesaplanması gerektiği anlaşılmıştır. Bu sebeple, BMH'nın hesaplanması hem kadın ve erkek için ayrı ayrı, hem de tüm veriler kullanılarak yapılmıştır.

\subsection{Sinyal önişleme (Signal preprocessing)}

ECG sinyali üzerinde oluşan gürültülerin temizlenmesi amaciyla sayısal filtre tasarlanmış ve uygulanmıştır. Gürülttü temizleme işlemi için 3 farklı filtre çeşidi kullanılmıştır. İlk önce IIR Chebyshev Type 2 Band Geçiren Filtre $(0.25-100 \mathrm{~Hz})$ kullanılmıştır (Şekil 2). Ardından IIR Chebyshev Type 2 Band Durduran Filtre (49-51 Hz) kullanılmıştır (Şekil 2). En son olarak ise gürültülerin en aza indirilmesi için Moving Filtresi uygulanarak gürültüler en aza indirilmiştir (Şekil 3).

Toplam 324 adet ECG verisini filtreleyerek bir sonraki adımda kullanmak için hazırlanmıştır. 


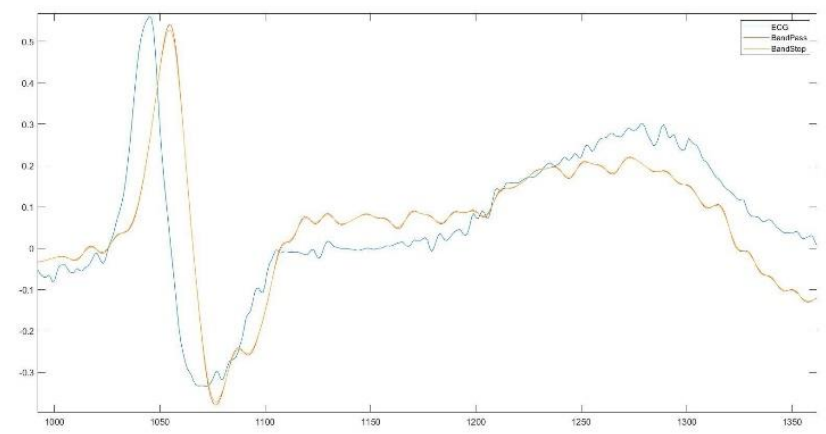

Şekil 2. Moving filtresi henüz uygulanmamış sinyal (Moving filter not yet applied signal)

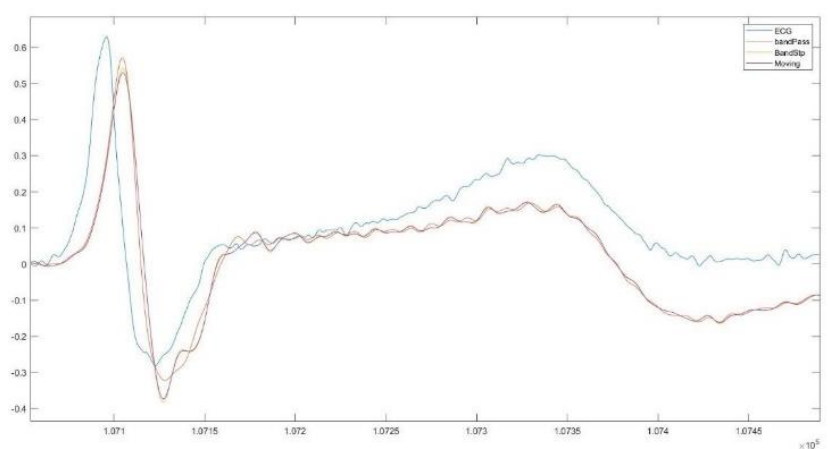

Şekil 3. Moving filtresi uygulanmış sinyal (Moving filter applied signal)

\section{3. Özellik çıkarımı (Feature extraction)}

Filtreleme işleminden sonra en iyi filtrelenmiş sinyaller dosyasını seçilmiştir. Seçilen dosyada her bir sinyal bir kişiye ait olan 324 sinyalin özelliklerini formüllerine göre hesaplayarak sinyallerin özellikleri çıkarılmıştır. $\mathrm{Bu}$ özelliklere ek olarak yapay zekanın bazal metabolizma hızını daha sağlıklı hesaplayabilmesi için bu özelliklere ek olarak kişilerin yaşı, boyu, kilosu gibi özellikleri de ekleyerek toplamda 23'ü formül hesaplamasıyla bulunan (Çizelge 1) sinyal özellikleri 4 tanesi kişisel özellik olmak üzere toplamda 27 özellik çıkarılarak özellik çıkarma işlemi sonlandırılmıştır.

\subsection{Spearman özellik seçme algoritması (Spearman feature selection algorithm)}

Orijinal veri setini temsil edebilecek en iyi altkümenin seçimi olarak tanımlanmaktadır. Özellik seçimi (diğer adıyla nitelik seçimi veya değişken seçimi), kullanılan algoritmaya göre özellikleri değerlendirerek veri setindeki $\mathrm{n}$ adet özellik arasından en iyi k adet özelliği seçme işlemidir (Forman 2003). Özellik seçimi, ilgilenilen problem için en faydalı ve en önemli özellikleri seçerek veri kümesindeki özellik sayısının azaltılmasını amaçlamaktadır. Bu çalışmada
Spearman korelasyon katsayısı tabanlı özellik seçme algoritması kullanılmışıtır.

Tablo 1. Sinyallerden çıkarılmış özellikler ve formülleri (Properties and formulas extracted from signals)

\begin{tabular}{|c|c|c|}
\hline No & Özellik & Formül \\
\hline 1 & Basıklık & $\frac{\sum_{i=1}^{n}(x(i)-\bar{x})^{4}}{(n-1) S^{4}}$ \\
\hline 2 & Çarpıklık & $\frac{\sum_{i=1}^{n}\left(x_{i}-\bar{x}\right)^{3}}{(n-1) S^{3}}$ \\
\hline 3 & IQR & $I Q R=i q r(x)$ \\
\hline 4 & $\mathrm{DK}$ & $D K=(S / \bar{x}) 100$ \\
\hline 5 & Geometrik Ortalama & $G=\sqrt[n]{x 1+\cdots+x n}$ \\
\hline 6 & Harmonik Ortalama & $H=n /\left(\frac{1}{x_{1}}+\cdots+\frac{1}{x_{n}}\right)$ \\
\hline 7 & $\begin{array}{l}\text { Activity- Hjort } \\
\text { Parameters }\end{array}$ & $A=S^{2}$ \\
\hline 8 & Maksimum & $x_{\max }=\max \left(x_{i}\right)$ \\
\hline 10 & $\begin{array}{l}\text { Ortalama Mutlak } \\
\text { Sapma }\end{array}$ & $M A D=\operatorname{mad}(x)$ \\
\hline 11 & Minimum & $x_{\min }=\min \left(x_{i}\right)$ \\
\hline 12 & Merkez Anlar & $C M=$ moment $(x, 10)$ \\
\hline 13 & Ortalama & $\begin{array}{r}\bar{x}=\frac{1}{n} \sum_{i=1}^{n}=\frac{1}{n}\left(x_{1}+\cdot \cdot\right. \\
\left.\cdot+x_{n}\right)\end{array}$ \\
\hline 14 & $\begin{array}{l}\text { Ortalama Eğri } \\
\text { Uzunluğu }\end{array}$ & $\mathrm{CL}=\frac{1}{n} \sum_{i=2}^{n}\left|x_{i}-x_{i-1}\right|$ \\
\hline 15 & Ortalama Enerji & $E=\frac{1}{n} \sum_{i=1}^{n} x_{i}^{2}$ \\
\hline 16 & $\begin{array}{l}\text { RMS-(Karekök } \\
\text { Ortalama) }\end{array}$ & $X_{r m s}=\sqrt{\frac{1}{n} \sum_{i=1}^{n}\left|x_{i}\right|^{2}}$ \\
\hline 17 & Standart Hata & $S \bar{x}=S / \sqrt{n}$ \\
\hline 18 & Standart Sapma & 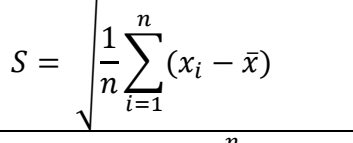 \\
\hline 19 & Şekil Faktörü & $S F=X_{r m s} /\left(\frac{1}{n} \sum_{i=1} \sqrt{\left|x_{i}\right|}\right)$ \\
\hline 20 & Tekil Değer Ayrışımı & $S V D=\operatorname{svd}(x)$ \\
\hline 21 & $\begin{array}{l}\% 25 \text { Kesilmiş } \\
\text { Ortalama }\end{array}$ & $T 25=\operatorname{trimmean}(x, 25)$ \\
\hline 22 & $\begin{array}{l}\% 50 \text { Kesilmiş } \\
\text { Ortalama }\end{array}$ & $T 50=\operatorname{trimmean}(x, 50)$ \\
\hline 23 & $\begin{array}{l}\text { Ortalama Teager } \\
\text { Enerjisi }\end{array}$ & $\begin{array}{l}\text { TE } \\
=\frac{1}{n} \sum_{i=3}^{n}\left(x_{i-1}^{2}-x_{i} x_{i-2}\right)\end{array}$ \\
\hline
\end{tabular}

İki sayısal ölçüm arasında doğrusal bir ilişki olup olmadığını, varsa bu ilişkinin yönünü ve şiddetinin ne olduğunu belirlemek için kullanılan bir istatistiksel yöntemdir. Bu sebeple çalışmamızda veri dağılımları normal dağılımdan uzak olduğundan Spearman 
Korelasyonu kullanılmaktadır. Yönteme ait denklemler şu şekilde özetlenebilir:

Çift Yönlü Test

$H 0: \rho s=0$

$H 1: \rho s \neq 0$

Test İstatistiği

$r_{s}=1-\frac{6 \Sigma d i^{2}}{n\left(n^{2}-1\right)}$

$d i=u i-v i$

$u i$ : 1. Örneklemdeki i. ölçümün sıra numarası

$v i$ : 2. Örneklemdeki i. ölçümün sıra numarası

Ret Bölgesi

$r s>r s, a / 2 \quad$ veya $\quad r s<-r s, a / 2 \quad$ ise $H 0$ reddedilir.

Denklemde elde edilen $r_{s}$ ilişki düzeni gösterir ve özellik seçiminde temel parametre olarak kullanılır. Her özelik için $r_{s}$ değeri hesaplandıktan sonra en iyi \%20 özellik seçilerek sistem tekrar modellenmiştir.

\subsection{Makine öğrenmesi (Machine learning)}

Makine öğrenmesi algoritmaları, kullandığ tekniklere göre denetimli ve denetimsiz öğrenme olmak üzere iki gruba ayrılırlar. Denetimsiz öğrenme, sadece giriş verilerinin olduğu bir öğrenme yöntemidir. Genellikle etiketsiz veriler üzerinde çalışılırken tercih edilen bir uygulamadır. Denetimli öğrenme ise giriş ve çıkış verilerinin ikisini de barındırmaktadır ve etiketlenmiş veriler kullanılarak öğrenme sağlanmaktadır. Giriş ve çıkış verileri bilindiği için öğrenme süreci denetlemeye tabi tutulur. $\mathrm{Bu}$ yüzden denetimli öğrenme olarak adlandırılmaktadır. Denetimli öğrenme, sınıflandırma ve regresyon olarak gruplandırılabilir. Regresyon, bir bağımlı değişken ile bağımsız değişkenler arasındaki ilişkiyi belirlemeye çalışan, nicel değişkenleri tahmin etmek için kullanılan bir denetimli öğrenmedir.Sınıflandırma ise tahmin edilen veriyi kategorilere atama ile ilgili bir denetimli öğrenmedir (Akay 2018).

$\mathrm{Bu}$ çalışmada kullanılan regresyon modelleri Lineer Regresyon, Gauss Süreç Regresyon, Destek Vektör Makineleri, Karar Ağaçları, Ensemble'dir. Bu sonuçlar çeşitli performans değerlendirme kriterleri göz ölüne alınarak karşılaştırılmıştır. Karşılaştırma sonucunda en iyi sonucu veren model belirlenmiştir.

Verilerin \%80'i eğitim, \%20'si test sürecinde kullanılmıştır.

\subsection{Performans değerlendirme kriterleri (Performance evaluation criteria)}

Çalışmada kullanılan performans değerlendirme kriteri olarak R², MSE, RMSE ve MAE kullanılmıştır. $\mathrm{Bu}$ performans kriterlerinden $\mathrm{R}^{2}$, modelin açıklayıcılık katsayısıdır. $\mathrm{Bu}$ katsayı, modelin tahmin ilişkisiyle doğru orantılıdır.
MSE, RMSE ve MAE birer hata ölçüleri olmaları sebebiyle modelin performansıyla ters orantılıdır. Yani düşük değerler, yüksek performansı göstermektedir (Wang and $\mathrm{Xu}$ 2004).

\subsubsection{Ortalama karesel hata (Mean squared error- $\mathrm{MSE})$}

MSE, modelin hatalarının karelerinin ortalamasını ifade eder. Tahmin edilen değerler gerçek değerlere ne kadar yaklaşırsa MSE değeri de o kadar küçülür.

$\mathrm{e}_{\mathrm{i}}$, gerçek değer $\left(\mathrm{y}_{\mathrm{i}}\right)$ ile tahmin edilen değer $\left(\widehat{\mathrm{y}}_{1}\right)$ arasındaki farktır.

$$
\begin{aligned}
& e_{i}=y_{i}-\widehat{y}_{l} \\
& M S E=\frac{1}{n} \sum_{i=1}^{n} e_{i}{ }^{2}
\end{aligned}
$$

\subsubsection{Ortalama karesel hata karekökü (Root Mean Squared Error-RMSE)}

RMSE, tahmin hatalarının ne kadar yayıldığının ölçüsüdür. MSE değerinin kareköküdür.

$R M S E=\sqrt{\frac{1}{n} \sum_{i=1}^{n} e_{i}^{2}}$

\subsubsection{Ortalama mutlak hata (Mean absolute error- MAE)}

MAE, veri kümesi içindeki her bir örnek için gerçek değer ile tahmin edilen değer arasındaki farkın mutlak değerinin ortalamasıdır.

$\operatorname{MAE}=\frac{1}{\mathrm{n}} \sum_{\mathrm{i}=1}^{\mathrm{n}}\left|\mathrm{e}_{\mathrm{i}}\right|$

\subsubsection{Açıklayıcılık katsayısı (Coefficient of determination)}

$\mathrm{R}^{2}$, bağımlı değişkendeki değişimin ne kadarının bağımsız değişken tarafından açıklanabildiğini gösteren bir katsayıdır. 1'e yakın olması beklenir.

$\mathrm{R}^{2}=1-\frac{\sum_{\mathrm{i}=1}^{\mathrm{n}}\left(\mathrm{y}_{\mathrm{i}}-\widehat{\mathrm{y}_{1}}\right)^{2}}{\sum_{\mathrm{i}=1}^{\mathrm{n}}\left(\mathrm{y}_{\mathrm{i}}-\overline{\mathrm{y}}\right)^{2}}$

\section{Sonuçlar (Conclusions)}

$\mathrm{Bu}$ çalışmada amaç ECG sinyali ile yapay zekayı kullanarak bireylere ait BMH değerini yüksek doğruluk oranında tahmin etmektir. Çalışmada izlenen yol şu şekildedir: İlk olarak bireylerden toplanan ECG sinyalleri filtrelenmiştir. Bu filtrelenmişs sinyallerden 23 adet özellik çıkarılmıştır ve demografik bilgiler eklenmiştir. Daha sonra kullanılacak özellikler özellik seçme algoritmaları yardımıyla belirlenmiştir. 
Belirlenen özellikler kullanılarak BMH tahmini için makine öğrenmesi algoritmaları kullanılmıştır. Son olarak modellerin karşılaştırılması için performans değerlendirme kriterleri kullanılmıştır.

Çalışmada, filtrelenmiş ECG sinyalinden özellikler çıkarılarak BMH tahmin edilmiştir. Bu tahminler ayrıca cinsiyet bazlı da yapılmıştır. Tahmin sonuçlarına bakıldığında, cinsiyet bazlı yapılmış tahminlerin daha iyi sonuç verdiği belirlenmiştir. Bütün veriler kullanıldığında (kadın-erkek) $\mathrm{R}=0.86$ bulunmuştur (Çizelge 4). Cinsiyet bazlı yapılan tahmin sonucunda erkek için $\mathrm{R}=0.85$ (Çizelge 2), kadınlarda ise $\mathrm{R}=0.98$ bulunmuştur (Çizelge 3). Buradaki sonuçlar göz önüne alındığında $\mathrm{BMH}$ tahminin cinsiyet bazlı tahminlerde daha iyi sonuçlar verdiği belirlenmiştir.
Sinyalden elde edilen bütün özellikler Spearman Özellik Seçme Algoritması kullanılarak azaltılmış ve BMH tahmin modeli hem cinsiyet bazlı hem de bütün veriler kullanılarak yeniden oluşturulmuştur. Cinsiyet gözetmeden yapılan tahmin modelinde $R=0.87$ başarı oranı elde edilirken (Çizelge 7), cinsiyet bazlı tahmin modelinde erkekler için $\mathrm{R}=0.91$ (Çizelge 5), kadınlar için $\mathrm{R}=0.99$ bulunmuştur (Çizelge 6). Özellik seçimi öncesi bulunan $\mathrm{R}$ değerleri ile özellik seçimi sonrası elde edilen $\mathrm{R}$ değerleri karşılaştırıldığında, özellik seçme algoritmalarının elde edilen başarı oranını arttırdığı belirlenmiştir.

Tablo 2. Erkekler için tüm özellikler kullanılarak yapılmış tahmin sonuçları (Estimation results using all features for men)

\begin{tabular}{llllll} 
Tüm Özellikler (Erkek) & RMSE & $\mathrm{R}^{2}$ & $\mathrm{MSE}$ & $\mathrm{MAE}$ & $\mathrm{R}$ \\
\hline Linear Regression (Interactions Linear) & - & - & - & - & - \\
\hline Linear Regression (Linear) & 82,88 & 0,73 & 6869 & 27,844 & 0,854 \\
\hline Linear Regression (Robust Linear) & 86,163 & 0,71 & 7424 & 21,133 & 0,842 \\
\hline SVM (Linear SVM) & 86,099 & 0,71 & 7413,1 & 29,29 & 0,842 \\
\hline Gaussian Process Regression (Squared Exponential GPR) & 87,775 & 0,69 & 7704,4 & 32,039 & 0,83 \\
\hline Gaussian Process Regression (Matern 5/2 GPR) & 88,196 & 0,69 & 7778,5 & 32,41 & 0,83 \\
\hline Gaussian Process Regression (Rational Quadratic GPR) & 87,775 & 0,69 & 7704,4 & 32,039 & 0,83 \\
\hline Stepwise Linear Regression & 90,349 & 0,68 & 8162,9 & 36,431 & 0,824 \\
\hline Gaussian Process Regression (Exponential GPR) & 89,297 & 0,68 & 7973,9 & 37,292 & 0,824 \\
\hline SVM (Quadratic SVM) & 93,936 & 0,65 & 8824 & 40,095 & 0,806 \\
\hline SVM (Medium Gaussian SVM) & 96,34 & 0,63 & 9281,4 & 52,088 & 0,793 \\
\hline SVM (Cubic SVM) & 100,06 & 0,6 & 10011 & 43,383 & 0,774 \\
\hline SVM (Coarse Gaussian SVM) & 109,41 & 0,52 & 11971 & 64,112 & 0,721 \\
\hline Ensemble (Boosted Trees) & 111,81 & 0,5 & 12502 & 79,813 & 0,707 \\
\hline Tree (Medium Tree) & 113,18 & 0,49 & 12810 & 48,804 & 0,7 \\
\hline Tree (Coarse Tree) & 118,43 & 0,44 & 14025 & 59,449 & 0,663 \\
\hline Tree (Fine Tree) & 120,51 & 0,42 & 14523 & 53,442 & 0,648 \\
\hline Ensemble (Bagged Trees) & 139,85 & 0,22 & 19558 & 113,52 & 0,469 \\
\hline SVM (Fine Gaussian SVM) & 151,17 & 0,09 & 22851 & 115,89 & 0,3 \\
\hline & & & & \\
\hline
\end{tabular}


Tablo 3. Kadınlar için tüm özellikler kullanılarak yapılmış tahmin sonuçları (Estimation results using all features for women)

\begin{tabular}{llllll} 
Tüm Özellikler (Kadin) & RMSE & $\mathrm{R}^{2}$ & MSE & MAE & $\mathrm{R}$ \\
\hline Linear Regression (Interactions Linear) & - & - & - & - & - \\
\hline Gaussian Process Regression (Squared Exponential GPR) & 25,977 & 0,98 & 674,8 & 21,297 & 0,989 \\
\hline Gaussian Process Regression (Rational Quadratic GPR) & 25,977 & 0,98 & 674,8 & 21,297 & 0,989 \\
\hline SVM (Linear SVM) & 29,69 & 0,97 & 881,52 & 21,266 & 0,984 \\
\hline Gaussian Process Regression (Matern 5/2 GPR) & 26,949 & 0,97 & 726,27 & 22,216 & 0,984 \\
\hline Linear Regression (Robust Linear) & 34,975 & 0,96 & 1223,3 & 23,162 & 0,979 \\
\hline Linear Regression (Linear) & 39,74 & 0,95 & 1579,3 & 31,505 & 0,974 \\
\hline Stepwise Linear Regression & 37,43 & 0,95 & 1401 & 29,285 & 0,974 \\
\hline SVM (Quadratic SVM) & 45,931 & 0,93 & 2109,6 & 35,588 & 0,964 \\
\hline Gaussian Process Regression (Exponential GPR) & 53,074 & 0,9 & 2816,8 & 38,396 & 0,948 \\
\hline SVM (Medium Gaussian SVM) & 83,709 & 0,76 & 7007,2 & 55,0731 & 0,871 \\
\hline Ensemble (Boosted Trees) & 87,375 & 0,74 & 7634,5 & 69,402 & 0,86 \\
\hline Tree (Fine Tree) & 96,378 & 0,68 & 9288,8 & 61,679 & 0,824 \\
\hline SVM (Coarse Gaussian SVM) & 97,32 & 0,67 & 9471,2 & 75,636 & 0,818 \\
\hline SVM (Cubic SVM) & 107,66 & 0,6 & 11590 & 67,981 & 0,774 \\
\hline Ensemble (Bagged Trees) & 119,73 & 0,5 & 14336,7 & 82,219 & 0,707 \\
\hline Tree (Medium Tree) & 133,39 & 0,38 & 17794 & 86,503 & 0,616 \\
\hline Tree (Coarse Tree) & 133,18 & 0,38 & 17736 & 94,504 & 0,616 \\
\hline SVM (Fine Gaussian SVM) & 155,64 & 0,16 & 24224 & 113,71 & 0,4
\end{tabular}

Tablo 4. Kadın - erkek için tüm özellikler kullanılarak yapılmış tahmin sonuçları (Estimation results using all features for men and women)

\begin{tabular}{llllll} 
Tüm Özellikler (Kadin-Erkek) & RMSE & $\mathrm{R}^{2}$ & $\mathrm{MSE}$ & $\mathrm{MAE}$ & $\mathrm{R}$ \\
\hline Linear Regression (Interactions Linear) & - & - & - & - & 0,86 \\
\hline Linear Regression (Linear) & 84,934 & 0,74 & 7213,8 & 63,327 \\
\hline Gaussian Process Regression (Squared Exponential GPR) & 84,774 & 0,74 & 7186,6 & 61,157 & 0,86 \\
\hline Gaussian Process Regression (Matern 5/2 GPR) & 84,585 & 0,74 & 7154,7 & 61,359 & 0,86 \\
\hline Gaussian Process Regression (Exponential GPR) & 85,275 & 0,74 & 7271,9 & 62,132 & 0,86 \\
\hline Gaussian Process Regression (Rational Quadratic GPR) & 84,774 & 0,74 & 7186,6 & 61,157 & 0,86 \\
\hline Linear Regression (Robust Linear) & 87,334 & 0,72 & 7627,2 & 65,576 & 0,848 \\
\hline Stepwise Linear Regression & 87,312 & 0,72 & 7623,4 & 67,402 & 0,848 \\
\hline SVM (Linear SVM) & 89,967 & 0,71 & 8094 & 64,769 & 0,842 \\
\hline SVM (Quadratic SVM) & 89,779 & 0,71 & 8060,3 & 67,073 & 0,842 \\
\hline Tree (Fine Tree) & 94,131 & 0,68 & 8860,7 & 68,348 & 0,824 \\
\hline SVM (Medium Gaussian SVM) & 100,17 & 0,64 & 10034 & 76,594 & 0,8 \\
\hline Tree (Medium Tree) & 101,17 & 0,63 & 10234 & 74,288 & 0,793 \\
\hline SVM (Coarse Gaussian SVM) & 106,13 & 0,59 & 11264 & 75,166 & 0,768 \\
\hline Ensemble (Bagged Trees) & 108,23 & 0,58 & 11713 & 79,183 & 0,761 \\
\hline Tree (Coarse Tree) & 115,19 & 0,52 & 13269 & 82,01 & 0,721 \\
\hline SVM (Cubic SVM) & 118,92 & 0,49 & 14141 & 89,597 & 0,7 \\
\hline Ensemble (Boosted Trees) & 120,28 & 0,48 & 14468 & 92,828 & 0,692 \\
\hline SVM (Fine Gaussian SVM) & 158,77 & 0,09 & 25207 & 119,8 & 0,3
\end{tabular}


Tablo 5. Erkekler için seçilmiş özellikler kullanılarak yapılmış tahmin sonuçları (Estimation results using selected features for men)

\begin{tabular}{llllll} 
Özellik Seçimi Sonrası (Erkek) & RMSE & $\mathrm{R}^{2}$ & MSE & MAE & R \\
\hline SVM (Cubic SVM) & - & - & - & - & - \\
\hline Linear Regression (Robust Linear) & 41,755 & 0,84 & 1743,5 & 19,106 & 0,916 \\
\hline SVM (Linear SVM) & 41,426 & 0,84 & 1716,1 & 18,553 & 0,916 \\
\hline SVM (Quadratic SVM) & 42,868 & 0,83 & 1837,7 & 21,616 & 0,911 \\
\hline SVM (Coarse Gaussian SVM) & 47,944 & 0,79 & 2298,7 & 26,141 & 0,888 \\
\hline Gaussian Process Regression (Exponential GPR) & 47,718 & 0,79 & 2277 & 27,251 & 0,888 \\
\hline SVM (Medium Gaussian SVM) & 49,063 & 0,78 & 2407,1 & 28,219 & 0,883 \\
\hline Gaussian Process Regression (Rational Quadratic GPR) & 49,964 & 0,77 & 2496,4 & 28,405 & 0,877 \\
\hline Tree (Medium Tree) & 51,241 & 0,76 & 2625,7 & 33,965 & 0,871 \\
\hline Gaussian Process Regression (Matern 5/2 GPR) & 51,174 & 0,76 & 2618,8 & 30,306 & 0,871 \\
\hline Linear Regression (Linear) & 52,191 & 0,75 & 2723,9 & 33,058 & 0,866 \\
\hline Linear Regression (Interactions Linear) & 52,516 & 0,74 & 2758 & 32,105 & 0,86 \\
\hline Stepwise Linear Regression & 52,517 & 0,74 & 2758 & 32,969 & 0,86 \\
\hline Gaussian Process Regression (Squared Exponential GPR) & 53,927 & 0,73 & 2908,1 & 32,678 & 0,854 \\
\hline Tree (Fine Tree) & 69,971 & 0,55 & 4854 & 44,723 & 0,741 \\
\hline Ensemble (Bagged Trees) & 70,754 & 0,53 & 5006,1 & 47,88 & 0,728 \\
\hline Tree (Coarse Tree) & 86,786 & 0,3 & 7531,7 & 69,151 & 0,547 \\
\hline SVM (Fine Gaussian SVM) & 86,482 & 0,3 & 7479,2 & 59,048 & 0,547 \\
\hline Ensemble (Boosted Trees) & 103,22 & 0,01 & 10655 & 86,678 & 0,1
\end{tabular}

Tablo 6. Kadınlar için seçilmiş özellikler kullanılarak yapılmış tahmin sonuçları (Estimation results using selected features for women)

\begin{tabular}{llllll}
\multicolumn{1}{c}{ Özellik Seçimi Sonrası (Kadın) } & RMSE & $\mathrm{R}^{2}$ & $\mathrm{MSE}$ & $\mathrm{MAE}$ & $\mathrm{R}$ \\
\hline Linear Regression (Interactions Linear) & - & - & - & - & - \\
\hline Linear Regression (Robust Linear) & 19,025 & 0,99 & 361,97 & 12,796 & 0,994 \\
\hline SVM (Linear SVM) & 17,309 & 0,99 & 299,6 & 11,796 & 0,994 \\
\hline Linear Regression (Linear) & 26,185 & 0,98 & 685,66 & 22,097 & 0,989 \\
\hline Gaussian Process Regression (Squared Exponential GPR) & 33,432 & 0,96 & 1117,7 & 26,242 & 0,979 \\
\hline Gaussian Process Regression (Matern 5/2 GPR) & 33,448 & 0,96 & 1118,7 & 26,077 & 0,979 \\
\hline Gaussian Process Regression (Rational Quadratic GPR) & 33,432 & 0,96 & 1117,7 & 26,242 & 0,979 \\
\hline SVM (Quadratic SVM) & 38,096 & 0,95 & 1451,3 & 28,163 & 0,974 \\
\hline Stepwise Linear Regression & 42,587 & 0,94 & 1813,6 & 29,613 & 0,969 \\
\hline Gaussian Process Regression (Exponential GPR) & 47,351 & 0,93 & 2242,1 & 33,013 & 0,964 \\
\hline SVM (Medium Gaussian SVM) & 71,894 & 0,83 & 5168,7 & 53,372 & 0,911 \\
\hline SVM (Cubic SVM) & 94,385 & 0,71 & 8908,6 & 58,26 & 0,842 \\
\hline Tree (Coarse Tree) & 97,264 & 0,7 & 9460,3 & 72,294 & 0,836 \\
\hline Ensemble (Boosted Trees) & 99,623 & 0,68 & 9924,8 & 79,491 & 0,824 \\
\hline Tree (Medium Tree) & 112,68 & 0,59 & 12697 & 81,429 & 0,768 \\
\hline SVM (Coarse Gaussian SVM) & 112,52 & 0,59 & 12662 & 89,811 & 0,768 \\
\hline Tree (Fine Tree) & 113,55 & 0,58 & 12893 & 76,206 & 0,761 \\
\hline Ensemble (Bagged Trees) & 118,06 & 0,55 & 13939 & 85,497 & 0,741 \\
\hline SVM (Fine Gaussian SVM) & 161,8 & 0,16 & 26180 & 124,83 & 0,4
\end{tabular}


Tablo 7. Kadın- erkek için seçilmiş özellikler kullanılarak yapılmış tahmin sonuçları (Estimation results using selected features for men and women)

\begin{tabular}{llllll} 
Özellik Seçimi Sonrası (Kadın-Erkek) & RMSE & $\mathrm{R}^{2}$ & $\mathrm{MSE}$ & $\mathrm{MAE}$ & $\mathrm{R}$ \\
\hline Linear Regression (Interactions Linear) & - & - & - & - & - \\
\hline Linear Regression (Linear) & 86,275 & 0,77 & 7443,4 & 63,878 & 0,877 \\
\hline Linear Regression (Robust Linear) & 85,866 & 0,77 & 7373 & 63,638 & 0,877 \\
\hline SVM (Linear SVM) & 85,128 & 0,77 & 7246,7 & 63,016 & 0,877 \\
\hline Gaussian Process Regression (Squared Exponential GPR) & 87,952 & 0,76 & 7735,6 & 65,767 & 0,871 \\
\hline Gaussian Process Regression (Matern 5/2 GPR) & 87,647 & 0,76 & 7682,1 & 65,916 & 0,871 \\
\hline Gaussian Process Regression (Rational Quadratic GPR) & 87,953 & 0,76 & 7735,6 & 65,767 & 0,871 \\
\hline Stepwise Linear Regression & 88,807 & 0,75 & 7886,7 & 67,225 & 0,866 \\
\hline Gaussian Process Regression (Exponential GPR) & 91,971 & 0,73 & 8458,7 & 65,723 & 0,854 \\
\hline SVM (Coarse Gaussian SVM) & 94,226 & 0,72 & 8878,6 & 68,319 & 0,848 \\
\hline SVM (Quadratic SVM) & 99,105 & 0,69 & 9821,8 & 71,61 & 0,83 \\
\hline Ensemble (Bagged Trees) & 107,56 & 0,64 & 11570 & 79,021 & 0,8 \\
\hline Tree (Fine Tree) & 108,3 & 0,63 & 11729 & 80,284 & 0,793 \\
\hline Tree (Medium Tree) & 107,74 & 0,63 & 11607 & 76,41 & 0,793 \\
\hline SVM (Medium Gaussian SVM) & 112,71 & 0,6 & 12704 & 74,559 & 0,774 \\
\hline Ensemble (Boosted Trees) & 115,53 & 0,58 & 13347 & 87,643 & 0,761 \\
\hline Tree (Coarse Tree) & 120,82 & 0,54 & 14598 & 84,6 & 0,734 \\
\hline SVM (Fine Gaussian SVM) & 157,97 & 0,21 & 24955 & 105,4 & 0,458 \\
\hline SVM (Cubic SVM) & 169,95 & 0,09 & 28882 & 94,595 & 0,3
\end{tabular}

Elde edilen sonuçlar göz önüne alındığında en iyi modeli belirlemek için performans değerlendirme kriterlerinden RMSE, MSE ve MAE değerlerinin 0'a yakın olması istenirken $\mathrm{R}$ ve $\mathrm{R} 2$ değerlerinin 1'e yakın olması istenmektedir. Özellik seçimi sonrası bulunan değerlere bakıldığında en iyi modelin Linear Regression modeli olduğu anlaşılmaktadır (Çizelge 5-6-7). ECG sinyali kullanılarak bir çalışma yapıldığında Linear Regression modeli cinsiyet bazlı uygulanırsa performans olumlu bir şekilde etkilenecektir.

\section{Tartışma ve Sonuç (Discussion and Conclusion)}

Araştırmada bulunan BMH değerleri ECG sinyalinin işlenmesiyle elde edilmiştir. Bireylerden toplanan ECG verileri işlenerek makine öğrenmesi algoritmaları yardımıyla BMH değerlerine ulaşılmıştır.

BMH günlük alınması gereken kalori miktarı hakkında en önemli yol göstericilerden biridir. Günümüzde BMH tespiti için kullanılan yöntemler oldukça zahmetli ve maliyetli olduğu için alternatif yöntemler aranmaktadır.

Literatürde BMH tespiti için genellikle dolaylı kalorimetre kullanılmaktadır (Arslan 1984). Dolaylı kalorimetre, kişinin oksijen alıp vermesini inceleyerek
BMH ölçümü yapmaktadır (LIU, LU, and CHEN 1995). Oluşturulan yeni tahmin modeli, literatürdeki yöntemle kıyaslandığında daha pratik ve maliyetsiz bir yöntem olduğu belirlenmiştir.

Dolaylı kalorimetre ile bulunan BMH değerlerinin doğru olabilmesi için deneklerin uyması gereken bazı koşullar vardır (12 saat aç kalınması, deney sırasında hareket edilmemesi, uyku düzeni vb.) (Tverskaya et al. 1998). Ancak oluşturulan yeni yöntemde herhangi bir koşul istenmemektedir. Bu da kişiden kaynaklı hataların minimuma indirilmesini sağlamaktadır. Bununla birlikte de yöntemin doğruluk oranı artmaktadır.

Sonuç olarak ECG sinyalleri ile BMH tahmini yapan bir sistem geliştirilmiş olup gündelik hayatta

kullanılabileceği düşünülmektedir. $\mathrm{Bu}$ sistemin avantajları zahmetsiz ve maliyetsiz olması, kişi kaynaklı hataları ortadan kaldırması ve ispatlanmış doğruluk oranı sayesinde güvenilir sonuçlar elde eden bir sistem olmasıdır. Tüm bu değerlendirmeler dikkate alındığında literatüre katkı sağlayacağı düşünülmektedir.

\section{Çıkar Çatıșması}

Yazar, bu makalenin yayınlanmasıyla ilgili herhangi bir çıkar çatışması olmadığını beyan eder. 


\section{Bilgilendirme}

\section{Finansal Destek}

Bu çalışma Sakarya Üniversitesi Bilimsel Araştırma Projeleri Komisyonu Tarafından Desteklenmiştir. Proje Numaras1: 2019-5-19-244.

\section{Etik Kurul Onayı}

$\mathrm{Bu}$ çalışmanın gerçekleştirilebilmesi için Sakarya Üniversitesi T1p Fakültesi'nden eti kurulu onayı alınmıștır. Onay tarih ve numarası 03/05/2019 71522473/050.01.04/112.

\section{Veri Kullanım İzni}

$\mathrm{Bu}$ çalışmanın gerçekleştirilebilmesi için Sakarya Üniversitesi Rektörlüğü'nden veri toplama ve kullanım izni alınmıştır. İzin tarih ve numarası 27/08/2019 35955870/604.02.

\section{Kaynaklar (References)}

Akay, Ebru Çağlayan. 2018. "Ekonometride Yeni Bir Ufuk: Büyük Veri ve Makine Öğrenmesi." Social Sciences Research Journal 7(2): 41-53.

Arslan, Perihan. 1984. "BAZAL METABOLİZMA HIZININ SAPTANMASINDA KULLANILAN YÖNTEMLERIN KIYASLANMASI VE BAZAL METABOLIZMA ENERJISININ VÜCUDUN YAĞSIZ DOKU KÜTLESİ İLE İLISSKISII ÜZERINDE BİR ARAŞTIRMA.” Beslenme ve Diyet Dergisi.

Ferrannini, Eleuterio. 1988. "The Theoretical Bases of Indirect Calorimetry: A Review.” Metabolism 37(3): 287-301.

Flancbaum, Louis et al. 1999. "Comparison of Indirect Calorimetry, the Fick Method, and Prediction Equations in Estimating the Energy Requirements of Critically Ill Patients." The American Journal of Clinical Nutrition 69(3): $\quad$ 461-66. https://academic.oup.com/ajcn/article/69/3/461/469417 9 (January 30, 2021).

Forman, George. 2003. 3 Journal of Machine Learning Research An Extensive Empirical Study of Feature Selection Metrics for Text Classification.

LIU, HSIU YING, YI FA LU, and WEI JAO CHEN. 1995. "Predictive Equations for Basal Metabolic Rate in Chinese Adults. A Cross-Validation Study." Journal of the American Dietetic Association 95(12): 1403-8.

Müller, B., S. Merk, U. Bürgi, and P. Diem. 2001. "Berechnung Des Grundumsatzes Bei Schwerer Und Morbider Adipositas." Praxis 90(45): 1955-63. https://europepmc.org/article/med/11817239 (January 30, 2021).

Nur Göz, Ayşe et al. 2017. EKG IŞSARETLERI YARDIMIYLA BIR OTOMATIKK ARITMI DEDEKTÖRÜ. Fatih Sultan Mehmet Vakıf Üniversitesi Biyomedikal Elektronik Tasarım, Uygulama ve Araștırma Merkezi. http://acikerisim.fsm.edu.tr:8080/xmlui/handle/11352/ 2491 (January 30, 2021).

Speakman, John R., Elzbieta Król, and Maria S. Johnson. 2004. "The Functional Significance of Individual Variation in Basal Metabolic Rate." In Physiological and Biochemical Zoology, The University of Chicago
Press

900-915. https://www.journals.uchicago.edu/doi/abs/10.1086/42 7059 (January 30, 2021).

Tverskaya, Rozalia, Russell Rising, Debra Brown, and Fima Lifshitz. 1998. "Comparison of Several Equations and Derivation of a New Equation for Calculating Basal Metabolic Rate in Obese Children." Journal of the American College of Nutrition 17(4): 333-36. https://pubmed.ncbi.nlm.nih.gov/9710841/ (January 30, 2021).

Wang, Wenjian, and Zongben Xu. 2004. "A Heuristic Training for Support Vector Regression." Neurocomputing 61(1-4): 259-75. 